\title{
Article \\ Comparing Patient Characteristics, Clinical Outcomes, and Biomarkers of Severe Asthma Patients in Taiwan
}

\author{
Shih-Lung Cheng ${ }^{1,2}$, Kuo-Chin Chiu ${ }^{3} \mathbb{D}$, Hsin-Kuo Ko ${ }^{4}$, Diahn-Warng Perng ${ }^{4}$, Hao-Chien Wang ${ }^{5}$, \\ Chong-Jen $\mathrm{Yu}^{5}{ }^{(\mathbb{D})}$, Chau-Chyun Sheu ${ }^{6}\left(\mathbb{D}\right.$, Sheng-Hao Lin ${ }^{7}$ and Ching-Hsiung Lin $7,8,9, *(\mathbb{D})$
}

1 Department of Internal Medicine, Far Eastern Memorial Hospital, Taipei 22260, Taiwan; shihlungcheng@gmail.com

2 Department of Chemical Engineering and Materials Science, Yuan Ze University, Zhongli, Taoyuan 320315, Taiwan

3 Division of Chest, Department of Internal Medicine, Luodong Poh-Ai Hospital, Yilan County 265, Taiwan; chiukc1@yahoo.com.tw

4 Department of Chest Medicine, Taipei Veterans General Hospital, Taipei 22260, Taiwan; kuohsink@ms67.hinet.net (H.-K.K.); dwperng@vghtpe.gov.tw (D.-W.P.)

5 Department of Internal Medicine, National Taiwan University Hospital and College of Medicine, National Taiwan University, Taipei 10617, Taiwan; haochienwang@gmail.com (H.-C.W.); jefferycjyu@ntu.edu.tw (C.-J.Y.)

6 Division of Pulmonary and Critical Care Medicine, Department of Internal Medicine, Kaohsiung Medical University Hospital, Kaohsiung Medical University, Kaohsiung City 80756, Taiwan; sheucc@gmail.com

7 Division of Chest Medicine, Department of Internal Medicine, Changhua Christian Hospital, Changhua 50006, Taiwan; shenghao@gmail.com

check for updates

Citation: Cheng, S.-L.; Chiu, K.-C.; Ko, H.-K.; Perng, D.-W.; Wang, H.-C.; Yu, C.-J.; Sheu, C.-C.; Lin, S.-H.; Lin, C.-H. Comparing Patient

Characteristics, Clinical Outcomes, and Biomarkers of Severe Asthma Patients in Taiwan. Biomedicines 2021, 9, 764. https://doi.org/10.3390/ biomedicines 9070764

Academic Editor: Girolamo Pelaia

Received: 18 May 2021

Accepted: 26 June 2021

Published: 1 July 2021

Publisher's Note: MDPI stays neutral with regard to jurisdictional claims in published maps and institutional affiliations.

Copyright: (c) 2021 by the authors. Licensee MDPI, Basel, Switzerland. This article is an open access article distributed under the terms and conditions of the Creative Commons Attribution (CC BY) license (https:/ / creativecommons.org/licenses/by/ $4.0 /)$.
8 Institute of Genomics and Bioformatics, National Chung Hsing University, Taichung 40227, Taiwan

9 Recreation and Holistic Wellness, MingDao Univeristy, Changhua 50006, Taiwan

* Correspondence: teddy@cch.org.tw; Tel.: +886-4-7238595; Fax: +886-4-7228289

Abstract: Purpose: To understand the association between biomarkers and exacerbations of severe asthma in adult patients in Taiwan. Materials and Methods: Demographic, clinical characteristics and biomarkers were retrospectively collected from the medical charts of severe asthma patients in six hospitals in Taiwan. Exacerbations were defined as those requiring asthma-specific emergency department visits/hospitalizations, or systemic steroids. Enrolled patients were divided into: (1) those with no exacerbations (non-exacerbators) and (2) those with one or more exacerbations (exacerbators). Receiver operating characteristic curves were used to determine the optimal cut-off value for biomarkers. Generalized linear models evaluated the association between exacerbation and biomarkers. Results: 132 patients were enrolled in the study with 80 non-exacerbators and 52 exacerbators. There was no significant difference in demographic and clinical characteristics between the two groups. Exacerbators had significantly higher eosinophils (EOS) counts (367.8 \pm 357.18 vs. $210.05 \pm 175.24, p=0.0043$ ) compared to non-exacerbators. The optimal cut-off values were 292 for EOS counts and 19 for the Fractional exhaled Nitric Oxide (FeNO) measure. Patients with an EOS count $\geq 300(\mathrm{RR}=1.88 ; 95 \% \mathrm{CI}, 1.26-2.81 ; p=0.002)$ or FeNO measure $\geq 20(\mathrm{RR}=2.10$; $95 \%$ CI, 1.05-4.18; $p=0.0356$ ) had a significantly higher risk of exacerbation. Moreover, patients with both an EOS count $\geq 300$ and FeNO measure $\geq 20$ had a significantly higher risk of exacerbation than those with lower EOS count or lower FeNO measure (RR $=2.16$; 95\% CI, 1.47-3.18, $p=<0.0001)$. Conclusions: Higher EOS counts and FeNO measures were associated with increased risk of exacerbation. These biomarkers may help physicians identify patients at risk of exacerbations and personalize treatment for asthma patients.

Keywords: asthma; eosinophil 


\section{Introduction}

Asthma is a heterogeneous disease with different phenotypes affecting children and adults worldwide [1,2]. The disease is chronic in its presentation, and characterized by inflammation and constriction of airways, wheezing, cough [2,3]. There are several phenotypes and endotypes with unique immunopathological mechanisms and diagnosis and symptoms often vary from patients to patients [4]. The World Health Organization estimates that asthma affects over 300 million people around the world [5].

The Global Initiative for Asthma (GINA) reports that asthma prevalence varies across the world, impacting 1-18\% of populations in different countries [2]. A nationwide survey of hospitalized patients in Taiwan found that the severity of asthma increases after 18 years of age [6]. Still, researchers generally agreed that prevalence of asthma in adults is generally lower than in western countries [7]. A time-trend analysis of asthma in Taiwanese adults found that the prevalence of asthma in 2011 was approximately $12 \%$ of those entered in the insurance system [8].

Asthma in adults is associated with various morbidities, creating higher utilization of healthcare systems. In countries such as Taiwan, governments must bear the financial burden of caring for adults with asthma-related disability through their healthcare systems [8]. A study by Sun et al. (2008) examined the healthcare utilization of asthma in Taiwanese adults by studying the healthcare utilization of the national health insurance system [9]. The mean costs of hospitalizations for patients with asthma were 2.7-fold higher than those for patients without asthma. [9]. There is also an associated quality of life decrease for patients with asthma.

The goal of treatment, as outlined by the 2020 GINA guidelines, is to control the symptoms of asthma and prevent any exacerbations. Initial treatment includes the use of short-acting bronchodilators and systemic corticosteroids [2]. In later, more severe patients, these medications may be increased in intensity. However, despite developments in medication, due to the heterogenous phenotypical nature of the disease, patients still suffer from unmanaged symptoms and remain at risk of exacerbations, needing severe medical care.

Biomarkers are measurable and reproducible biological processes that often correlate with the "clinical state" of a patient [10]. Biomarkers are particularly important for respiratory diseases to understand the best treatment options and predict the progression of the disease [3]. Biomarkers have been previously used to understand personalized treatments for patients that might benefit from asthma treatment [11]. With the growing interest in precision medicine, biomarkers are already starting to be used by clinicians in the identification and treatment of patients for a variety of other diseases [1]. For asthma, however, biomarkers have not yet been commonly used to identify and track disease progression and treatment [1].

Recent studies have shown that some traits can predict future exacerbation, which includes past exacerbation history, asthma control and type 2 inflammation. Type 2 inflammation is found in approximately $50-70 \%$ of severe asthma patients $[12,13]$ and is characterized by the accumulation of Th2 cells [14]. Immunoglobin E (IgE), fractional exhaled nitric oxide (FeNO) and blood or sputum eosinophil (EOS) are recognized biomarkers used for the identification of type 2 inflammation [2]. Knowing these biomarkers for adult asthma would help in predicting treatment effectiveness, disease prognosis, the associated healthcare resources utilizations, and costs for patients in Taiwan. This is particularly significant given that previous lack of data for adults with asthma. The goal of this study is to understand the demographic and clinical characteristics of severe asthma patients in Taiwan. Additionally, this analysis also intends to understand the association between biomarkers and exacerbations of asthma in adults. 


\section{Materials and Methods}

\subsection{Study Design and Setting}

This is a multicenter retrospective cohort study to compare patient characteristics, clinical outcomes and biomarkers for severe asthma patients in Taiwan. The severe asthma patients were enrolled in outpatient visits in six hospitals during 2016-2019, including Far Eastern Memorial Hospital, National Taiwan University Hospital, Taipei Veterans General Hospital, Kaohsiung Medical University Hospital, Changhua Christian Hospital and Poh-Ai Hospital. (approval number: FEMH: IRB No. 105131-F) and conducted in accordance with the Declaration of Helsinki. All patients provided written informed consent for participation, and the study was registered at https:/ /www.clinicaltrials. gov (NCT02871947) on 18 August 2016. Informed consent was obtained from all subjects involved in the study.

\subsection{Study Population}

\subsubsection{Inclusion Criteria}

All patients with more than 1 year follow up in outpatient visits were enrolled. Patients requiring medium to high dose ICS and LABA or leukotriene modifier/theophylline for the previous year, or those treated with systemic corticosteroids for more than half a year to prevent from becoming uncontrolled, or those remaining uncontrolled despite this therapy were further included in the study as severe asthma patients. Patients fulfilling at least one of the following criteria are defined as uncontrolled asthma: (1) having ACQ score $>1.5$ or ACT score $<19$, (2) treated with two or more bursts of systemic corticosteroids, (3) having at least one hospitalization, ICU stay or mechanical ventilation in the previous year, (4) having FEV $1<80 \%$ predicted after appropriate bronchodilator withheld and with reduced FEV1/FVC.

\subsubsection{Exclusion Criteria}

Patients with COPD or other pulmonary disease, with lung or other end-stage cancers, with oxygen therapy for more than $15 \mathrm{~h}$ a day, with long-term use of non-invasive positive pressure ventilation (NIPPV), with active tuberculosis disease or other infection or without agreement were excluded.

\subsection{Data Collection and Outcome Measurement}

We have collected the following information for 1 year from medical charts: age, gender, BMI, smoking history, pre-existing comorbidities, lung function, biomarker data, treatment and frequency of serious exacerbations. Pre-existing comorbidities contained allergic rhinitis, nasal polyps, hypertension, gastroesophageal reflux disease (GERD), heart failure (HF), diabetes mellitus (DM), and atopic history. At the time of enrollment, the participants were assessed for Asthma Control Test (ACT), lung function and biomarker data including IL-5, IL-13, IL-8, IL-17, IL-33, Tryptase, Periostin, TGF Beta, TNF Alpha, vascular endothelial growth factor (VEGF), placenta growth factor (PIGF), TSLP, eosinophil (EOS) count, and fractional exhaled nitric oxide (FeNO) and Immunoglobulin E (IgE) level.

Serious exacerbations were evaluated in the current study and defined as requiring asthma-specific emergency department visits or hospitalization, or systemic steroids after entering the database.

\subsection{Statistical Analyses}

The demographic and clinical characteristics of the study subjects were described. Continuous variables were presented as mean \pm standard deviation, and the difference between groups was evaluated using the independent $t$ test. Categorical variables were presented as numbers and percentages, and the chi-squared or Fisher's exact test was used to evaluate the difference between groups. The receiver operating characteristic (ROC) curves were plotted to determine the optimal cut-off point value for EOS counts and FeNO measure. The optimal cut-point value was then obtained using the maximum value of 
Youden's index in ROC analysis. The association between exacerbation and biomarkers was analyzed by the generalized linear model and presented using the forest plot. The $p$ values less than 0.05 were considered statistically significant. All analyses were performed using the SAS/Stat system for Windows, version 9.3 (SAS Institute, Cary, NC, USA).

\section{Results}

The demographic and clinical characteristics of enrolled patients are described in Table 1. A total of 132 patients were enrolled in the study. The average age of all patients was $61.47 \pm 13.12$ years, and $82(62.12 \%)$ patients were female. The average BMI was $25.43 \pm 4.83$, suggesting enrolled patients being overweight. In terms of pre-existing comorbidities, more than 70 percent of patients had allergic rhinitis, while less than 10 percent of patients had nasal polyps and heart failure. There were 0.73 exacerbations per year. Of 132 patients, there were $52(39.39 \%)$ patients with one or more exacerbations (exacerbators) and $80(60.61 \%)$ patients with no exacerbations (non-exacerbators). Comparing exacerbators and non-exacerbators, there was no significant difference in demographic and clinical characteristics. The detailed medications and biomarkers of severe asthma patients were in Table S1.

Table 1. Demographic and clinical characteristic of severe asthma patients.

\begin{tabular}{|c|c|c|c|c|}
\hline & All Patients & No Exacerbations & Exacerbations $\geq 1$ & \\
\hline & $(n=132)$ & $(n=80)$ & $(n=52)$ & $p$-Value \\
\hline & $n(\%)$ & $n(\%)$ & $n(\%)$ & \\
\hline Age, mean $\pm S D$ & $61.47 \pm 13.12$ & $62.19 \pm 13.39$ & $60.37 \pm 12.74$ & 0.4377 \\
\hline Gender & & & & 0.7980 \\
\hline Male & $50(37.88)$ & $31(38.75)$ & $19(36.54)$ & \\
\hline Female & $82(62.12)$ & $49(61.25)$ & $33(63.46)$ & \\
\hline $\mathrm{BMI}$, mean $\pm \mathrm{SD}$ & $25.43 \pm 4.83$ & $25 \pm 4.74$ & $26.07 \pm 4.95$ & 0.2150 \\
\hline Smoking History & $34(25.76)$ & $19(23.75)$ & $15(28.85)$ & 0.5130 \\
\hline Allergic Rhinitis (AR) & 95 (71.97) & $60(75)$ & $35(67.31)$ & 0.3363 \\
\hline Nasal Polyps & $4(3.03)$ & $2(2.5)$ & $2(3.85)$ & 0.6464 \\
\hline Hypertension & $60(45.45)$ & $35(43.75)$ & $25(48.08)$ & 0.6257 \\
\hline GERD & $23(17.42)$ & $13(16.25)$ & $10(19.23)$ & 0.6591 \\
\hline Heart Failure (HF) & $12(9.09)$ & $7(8.75)$ & $5(9.62)$ & 1.0000 \\
\hline $\mathrm{DM}$ & $27(20.45)$ & $17(21.25)$ & $10(19.23)$ & 0.7787 \\
\hline Atopic & $72(54.55)$ & $49(61.25)$ & $23(44.23)$ & 0.0550 \\
\hline FEV1, mean \pm SD & $1.54 \pm 0.7$ & $1.54 \pm 0.7$ & $1.55 \pm 0.71$ & 0.9408 \\
\hline FEV $1 \%$, mean $\pm S D$ & $66.93 \pm 23.51$ & $68.2 \pm 23.98$ & $64.97 \pm 22.85$ & 0.4425 \\
\hline FEV1/FVC, mean \pm SD & $66.86 \pm 12.14$ & $66.82 \pm 12.8$ & $66.93 \pm 11.18$ & 0.9599 \\
\hline Exacerbation(s), mean $\pm \mathrm{SD}$ & $0.73 \pm 1.35$ & & & \\
\hline
\end{tabular}

Patient biomarker data are presented in Table 2. The average Eosinophils count was $272.67 \pm 272.91$, FeNO Measure was $34.12 \pm 23.08$ and IgE level was $241.26 \pm 331.20$. As compared with non-exacerbators, exacerbators had significantly higher eosinophils counts (367.8 \pm 357.18 vs. $210.05 \pm 175.24, p=0.0043)$ and TSLP levels $(17.16 \pm 13.93$ vs. $11.59 \pm 14.15, p=0.0166)$, but significantly lower tryptase levels (1283.41 $\pm 1480.97 \mathrm{vs}$ $2270.33 \pm 3228.43, p=0.0191$ ). 
Table 2. Biomarkers of severe asthma patients.

\begin{tabular}{|c|c|c|c|c|}
\hline & All Patients & No Exacerbations & Exacerbations $\geq 1$ & \multirow{3}{*}{$p$-Value } \\
\hline & $(n=132)$ & $(n=80)$ & $(n=52)$ & \\
\hline & Mean \pm SD & Mean \pm SD & Mean \pm SD & \\
\hline IL-5 & $2.42 \pm 1.58$ & $2.35 \pm 1.37$ & $2.53 \pm 1.87$ & 0.5603 \\
\hline IL-13 & $59.37 \pm 28.32$ & $61.33 \pm 25.7$ & $56.39 \pm 31.92$ & 0.3528 \\
\hline Tryptase & $1881.54 \pm 2715.44$ & $2270.33 \pm 3228.42$ & $1283.41 \pm 1480.97$ & 0.0191 \\
\hline Periostin & $16.26 \pm 10.43$ & $15.56 \pm 10.13$ & $17.36 \pm 10.9$ & 0.3396 \\
\hline IL-8 & $15.94 \pm 52.69$ & $18.81 \pm 66.55$ & $11.41 \pm 12.99$ & 0.3403 \\
\hline IL-17 & $12.59 \pm 4.4$ & $12.68 \pm 4.8$ & $12.44 \pm 3.76$ & 0.7565 \\
\hline TGF Beta & $30.81 \pm 23.56$ & $28.25 \pm 15.3$ & $34.86 \pm 32.4$ & 0.1816 \\
\hline TNF Alpha & $6.17 \pm 18.95$ & $4.5 \pm 11.23$ & $8.87 \pm 27.08$ & 0.2865 \\
\hline VEGF & $338.67 \pm 296.96$ & $346.33 \pm 333.07$ & $327.04 \pm 234.27$ & 0.6980 \\
\hline PIGF & $7.31 \pm 6.7$ & $7.85 \pm 7.23$ & $6.47 \pm 5.74$ & 0.2563 \\
\hline TSLP & $13.98 \pm 14.33$ & $11.59 \pm 14.15$ & $17.76 \pm 13.93$ & 0.0166 \\
\hline IL-33 & $3.87 \pm 4.24$ & $4.01 \pm 4.96$ & $3.67 \pm 2.85$ & 0.6191 \\
\hline EOS & $272.67 \pm 272.91$ & $210.05 \pm 175.24$ & $367.8 \pm 357.18$ & 0.0043 \\
\hline $\mathrm{FeNO}$ & $34.12 \pm 23.08$ & $32.77 \pm 22.99$ & $36.16 \pm 23.29$ & 0.4190 \\
\hline IgE Levels & $241.26 \pm 331.20$ & $239.13 \pm 321.43$ & $244.52 \pm 348.84$ & 0.9283 \\
\hline
\end{tabular}

The optimal cut-off points for EOS and FeNO were determined by the ROC curves (Figure 1). The optimal cut-off point values were 291.76 for EOS counts and 19 for FeNO measure. We then selected 300 as the cut-off point for EOS counts and 20 for FeNO measure. There were 90 patients with EOS count $<300$ and 41 patients with EOS count $\geq 300$, while there were 32 patients with FeNO measure $<20$ and 96 patients with FeNO measure $\geq 20$. For IgE Level, there were 63 patients with IgE level $<100$ and 66 patients with IgE level $\geq 100$. As compared with non-exacerbators, exacerbators had a higher percentage of patients with EOS count $\geq 300(46.15 \%$ vs. $21.52 \%, p=0.0029)$ or with FeNO measure $\geq 20$ ( $86.27 \%$ vs. $67.53 \%, p=0.0165)$; however, there was no significant difference in IgE level between exacerbators and non-exacerbators (Table 3). Moreover, we found that those with EOS $\geq 300$ and $\mathrm{FeNO} \geq 20$ were more commonly treated with certain medications (with ICS/LABA/LAMA, LTA, omalizumab and prednisolone).

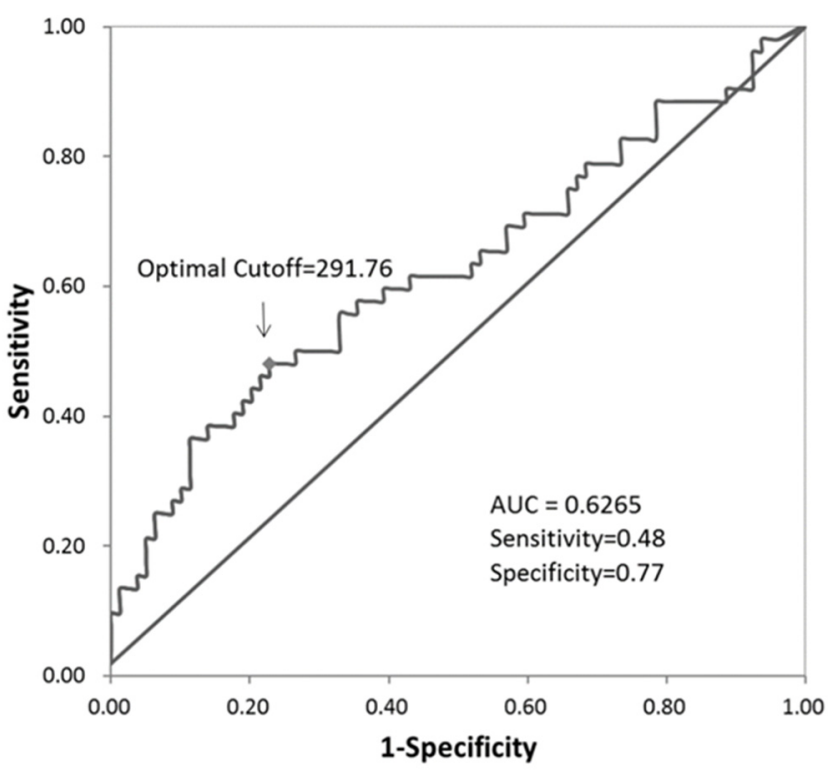

(A)

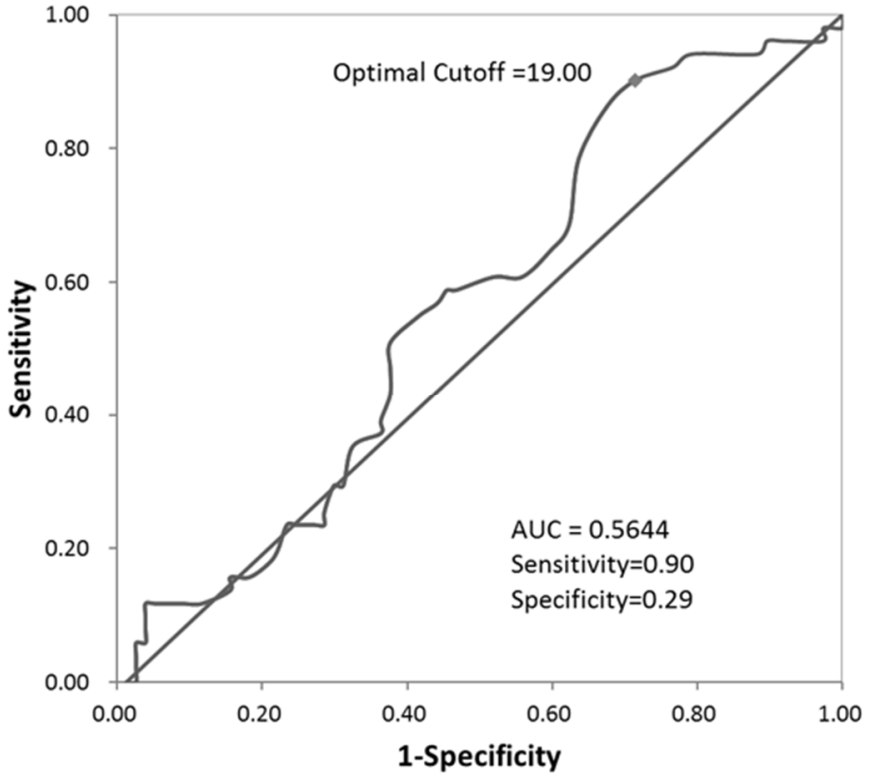

(B)

Figure 1. The ROC curves for the optimal cut-off point value for Eosinophils count (A) and FeNo measure (B). 
Table 3. The relationships between exacerbation and biomarkers.

\begin{tabular}{|c|c|c|c|c|}
\hline & All Patients & No Exacerbations & Exacerbations $\geq 1$ & \\
\hline & $(n=132)$ & $(n=80)$ & $(n=52)$ & $p$-Value \\
\hline & $n(\%)$ & $n(\%)$ & $n(\%)$ & \\
\hline Eosinophils Count & & & & 0.0029 \\
\hline$<300$ & $90(68.70)$ & $62(78.48)$ & $28(53.85)$ & \\
\hline$\geq 300$ & $41(31.30)$ & $17(21.52)$ & $24(46.15)$ & \\
\hline missing & 1 & 1 & & \\
\hline FeNO Measure & & & & 0.0165 \\
\hline$<20$ & $32(25.00)$ & 25 (32.47) & $7(13.73)$ & \\
\hline$\geq 20$ & $96(75.00)$ & $52(67.53)$ & $44(86.27)$ & \\
\hline missing & 4 & 3 & 1 & \\
\hline IgE Levels & & & & 0.9733 \\
\hline$<100$ & $63(48.84)$ & $38(48.72)$ & $25(49.02)$ & \\
\hline$\geq 100$ & $66(51.16)$ & $40(51.28)$ & $26(50.98)$ & \\
\hline missing & 3 & 2 & 1 & \\
\hline
\end{tabular}

Since both EOS count and FeNO measure showed a statically significant relationship with exacerbation, we further combined EOS count and FeNO measure as a biomarker group to evaluate its relationship with exacerbation. There were 26 patients with EOS count $<300$ and FeNo measure $<20,6$ patients with EOS count $\geq 300$ and FeNO measure $<20,62$ patients with EOS count $<300$ and FeNo measure $\geq 20$, and 33 patients with EOS count $\geq 300$ and FeNO measure $\geq 20$. As compared with nonexacerbators, exacerbators had a higher percentage of patients with EOS count $\geq 300$ and FeNO measure $\geq 20(43.14 \%$ vs. $14.47 \%, p=0.0019)$ (Table 4$)$.

Table 4. The relationships between exacerbation and the biomarker group.

\begin{tabular}{|c|c|c|c|c|}
\hline & All Patients & No Exacerbations & Exacerbations $\geq 1$ & \\
\hline & $(n=132)$ & $(n=80)$ & $(n=52)$ & $p$-Value \\
\hline & $n(\%)$ & $n(\%)$ & $n(\%)$ & \\
\hline Biomarker group \#1 & & & & 0.0019 \\
\hline EOS $<300 \&$ FeNO $<20$ & $26(20.47)$ & $20(26.32)$ & $6(11.76)$ & \\
\hline $\mathrm{EOS} \geq 300 \& \mathrm{FeNO}<20$ & $6(4.72)$ & $5(6.58)$ & $1(1.96)$ & \\
\hline $\mathrm{EOS}<300 \& \mathrm{FeNO} \geq 20$ & $62(48.82)$ & $40(52.63)$ & $22(43.14)$ & \\
\hline $\begin{array}{c}\mathrm{EOS} \geq 300 \& \mathrm{FeNO} \geq 20 \\
\text { Missing }\end{array}$ & $33(25.98)$ & $11(14.47)$ & $22(43.14)$ & \\
\hline Biomarker group \#2 & & & & $<0.0001$ \\
\hline $\mathrm{EOS} \geq 300 \& \mathrm{FeNO} \geq 20$ & $33(20.47)$ & $11(14.47)$ & $22(43.14)$ & \\
\hline Others & $94(79.53)$ & $65(85.53)$ & $29(56.86)$ & \\
\hline Missing & 5 & 4 & 1 & \\
\hline
\end{tabular}

The generalized linear models demonstrated a significant association between the biomarker group and exacerbation (Figure 2). Patients with EOS count $\geq 300$ and FeNO measure $\geq 20$ were associated with the worst outcome $(R R=2.16 ; 95 \%$ CI, $1.47-3.18$; $p=<0.0001)$. 


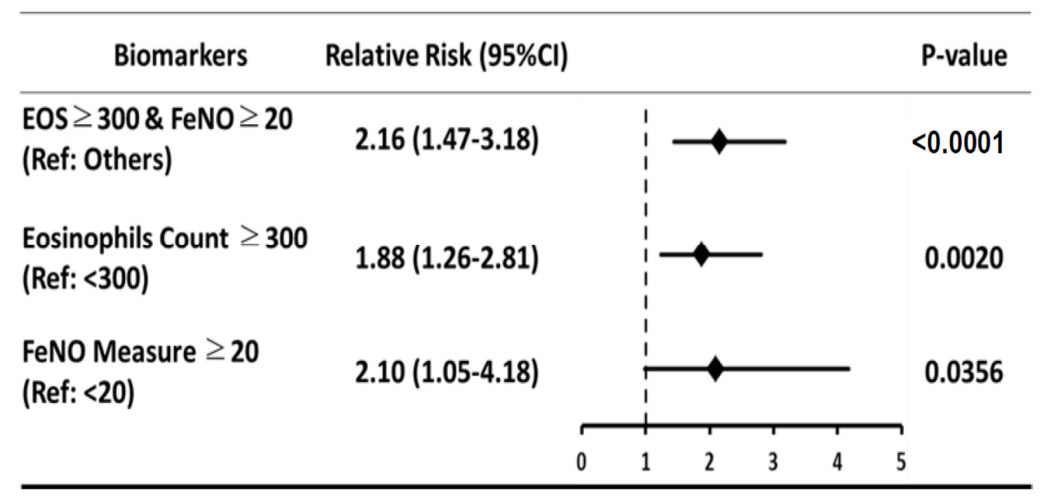

Figure 2. The association between Exacerbation and Biomarkers.

\section{Discussion}

We conducted a retrospective review study in Taiwan to analyze the clinical and demographic characteristics of asthma patients and the association between biomarkers and number of asthma exacerbations, compared with our optimal cut-off point. We found that patients with higher EOS and FeNO levels corresponded with a higher number of exacerbations. Additionally, the combination of the two biomarkers EOS count $\geq 300$ and FeNO measure $\geq 20$ was significantly associated with more exacerbations compared to those with a lower level of EOS and FeNO. We concentrated on these biomarkers, considering that the GINA guidelines note that FeNO is a useful biomarker for type 2 asthma, identifies airway inflammation, and is related to levels of blood eosinophils [2]. This study defines an optimal cut off point for biomarkers and highlights the need to use biomarkers in personalized medicine to improve treatment for asthma patients.

A cross-sectional matched cohort study by Price et al. (2019), used real-world evidence to understand biomarkers in predicting asthma exacerbations for patients using inhaled corticosteroids, and validates the findings of our own study [15]. The study used data from Optum Patient Care Research database (OPRC) to categorize patients based on their FeNO and EOS levels, and two different cut-off points. Price et al. (2019) found that those with high FeNO levels ( $\geq 50 \mathrm{ppb})$ and high blood eosinophil levels $\left(\geq 0.300 \times 10^{9}\right.$ cells $\left./ \mathrm{L}\right)$ were more likely to have an increased rate of exacerbations (RR: 3.67$)$ compared their matched counterparts [15]. As their study pointed out, EOS and FeNO are potentially simple biomarkers to measure, and may help targeted medication for asthma patients [15]. A study by Saito et al. (2014), found that the close monitoring of FeNO levels predicted asthma exacerbations in patients with uncontrolled disease [16]. Studies like these identify the benefits of personalized treatments and monitoring. Our study identified that the optimal cut off point values were 292 for EOS and 19 for FeNo. A systematic literature review conducted by the Agency for Healthcare Research and Quality examined various cut off points for diagnosing asthma. After reviewing 43 studies, researchers found that for cut off levels of $<20 \mathrm{ppb}$ and $20-30 \mathrm{ppb}$, FeNO testing had a sensitivity of 0.79 and 0.64 , respectively, and specificity of 0.72 and 0.81 , respectively [17]. While this fits with our own cut-off points, additional research might help improve correctly using the biomarkers FeNO and identify a cut off level that can be used by all healthcare providers.

While we studied the utility of lung function in predicting exacerbation (FEV1), our study found no correlation between the two factors. In contrast, a Swedish study by Malinovschi et al. (2016), looked at the relationship between FeNO and EOS levels and lung function [18]. This study did find a relationship between biomarkers and lung function. They noted that patients with higher FeNO levels and higher EOS counts were associated having obstructed airways (FEV1). However, their study used a FeNO cut off of 20-25 ppb and $0.3 \times 109 /$ L for blood eosinophil counts (based on age) [18]. A potential reason why we did not see an association might be our more conservative cut off point.

Additionally, while we examined the roll of the blood biomarker IgE, we found no association between exacerbations and $\operatorname{IgE}$. IgE, like FEV is associated with "lower lung 
function" and higher levels of "allergen-specific IgE are associated with risk of asthma [19]." However, IgE may be difficult to measure, as it has a short half-life compared to other "immunoglobulin isotopes" $[19,20]$ and low sensitivity/specificity "for detecting sputum eosinophilia" $[19,21]$. In our study, there may have been a relationship between IgE and exacerbations, but we did not detect it.

According to the 2020 GINA report, type 2 inflammation in asthma is common in approximately $50 \%$ of patients with asthma. It is "characterized by various cytokines ((IL)-4, IL-5, and IL-3))", and that are produced in "adaptive immune system on recognition of allergens" [2]. Type 2 inflammation often uses biomarkers to identify mild and moderate asthma. GINA reports that type 2 inflammation is characterized by "eosinophilia or increase FeNO, and may be accompanied by atopy, whereas non-Type 2 inflammation is often characterized by increased neutrophils." [2].

While we did not group patients into type 1 and type 2 inflammation categories, we did look at treatment behavior among sub-groups (Supplementary Table S1). We found that those with EOS $\geq 300$ and $\mathrm{FeNO} \geq 20$ were more commonly treated with certain medications (with ICS/LABA/LAMA, LTA, omalizumab and prednisolone), but that outcomes (number of exacerbations) of these patients were not impacted by these treatments. A potential explanation is that these patients may have "poor sensitivity" to these medications [15]. Their EOS and FeNO levels remained high despite their medication regime. This may, in turn, indicate a need for more targeted/personalized treatment that lowers their risk of future exacerbations [15]. Biologics that use the pathway for anti-interleukin (like anti IL-4R and anti IL5) can potentially reduce the exacerbations and decrease inflammatory biomarkers. Researchers have already suggested that these biomarkers can guide biologics treatment [22,23].

Higher efficiency biologics are correlated with higher levels of FeNO and EOS two other seminal studies. A trial by Castro et al. (2018) compared an anti-IL4 inhibitor (dupilumab) at various dosages with a placebo. They found that asthma exacerbations were lower among patients who received the biologic drug, approximately $48 \%$ lower for those who received either $200 \mathrm{mg}$ or $300 \mathrm{mg}$. Patients who took this drug also had higher FEV1. The higher efficacy in terms of reducing exacerbations and improving lung function are correlated with higher baseline levels of FeNO (>25 ppm) and EOS counts ( $>300$ cells per $\mu \mathrm{L}$ ) [24]. Another study by Ortega et al. (2016) studied the impact of the biologic drug mepolizumab on eosinophilic asthma. The researchers found that the biologic drug reduced the average number of exacerbations per person per year from approximately 2 to 1 when compared to placebo. They highlighted that higher baseline counts of EOS ( $>150$ cells per $\mu \mathrm{L}$ ) in patients translated to more greater reductions in exacerbation with the use of drug [25]. The Castro study emphasized the efficacy of antiIL4 agent and the Ortega study is a post-hoc analysis of data for MENSA and DREAM study for mepolizumab. These studies, similar to our study, found that patients with higher EOS and FeNO biomarkers would have more exacerbation rates, though the cut levels of these biomarkers were different. These biologics present a way forward in treatment, as patients who use them may drastically improve their quality of life.

In our study, another marker is tryptase. Patients with severe asthma with high blood eosinophil counts and low serum tryptase levels were more likely to have greater risk of exacerbation. This result was reported in our previous study [26]. The gene expression of mast cell tryptase is increased in asthmatic epithelium, especially in the type 2-high subgroup, and predicts the responsiveness to ICS [27]. The numbers of airway tissue mast cells and the concentration of bronchoalveolar lavage tryptase can determine the efficacy of ICS treatment in persistent asthma [28]. The findings of our study indicating low levels of tryptase associated with a higher risk of exacerbation implied that lower levels of serum tryptase may be linked to non-allergic type 2 inflammation or non-type 2 inflammation (ILC2-related or neutrophilic inflammation). Therefore, lower levels of serum tryptase are potentially corticosteroid-resistant and refractory to ICS/LABA treatment and associated with high risk of asthma exacerbation. 
This study had several limitations. We did not control for baseline differences between the exacerbations and no exacerbations groups. Therefore, there is no matching of the cohort on underlying comorbidities, patient covaries, or baseline treatments. However, this reflects the real application of our study, considering that not all patients who have asthma will also have the same underlying conditions. Follow up for our study was only limited to one year. A longer follow up period would allow us to see long-term effects.

\section{Conclusions}

Our study demonstrated that higher EOS counts and FeNO measure were associated with an increased risk of exacerbation. Identification of these biomarkers may help physicians identify patients at risk of exacerbations and personalize treatment for asthma patients. In the future, because of the heterogenous nature of asthma in adults, the identification of biomarkers that put adults at risk of increased exacerbations may help with treatment selection by clinicians. In the future, payers and HTAs will seek the most relevant populations to make decisions about coverage with various medications. Additional studies might examine outcomes (reduction in exacerbations) based on different treatments once the patients have been identified in terms of biomarkers.

Supplementary Materials: The following are available online at https://www.mdpi.com/article/10 $.3390 /$ biomedicines $9070764 /$ s1.

Author Contributions: Methodology, S.-L.C., K.-C.C.; Software: C.-C.S.; Validation, H.-K.K.; Formal Analysis: S.-H.L., C.-H.L.; Writing-Original: S.-L.C., K.-C.C.; Draft Preparation: S.-L.C., K.-C.C.; Writing-Review and Editing, D.-W.P., H.-C.W., C.-J.Y., C.-H.L.; Visualization, C.-H.L.; Supervision, C.-H.L. All authors have read and agreed to the published version of the manuscript.

Funding: This research received no external funding.

Institutional Review Board Statement: The study was conducted according to the guidelines of the Declaration of Helsinki, and approved by the Institutional Review Board of Far Eastern Memorial Hospital (IRB No. 105131-F) in 2016.

Informed Consent Statement: Informed consent was obtained from all subjects involved in the study.

Data Availability Statement: The data will not be shared with a reason.

Acknowledgments: We would like to thank Bruce Wang, Po-Ya Chuang, and Ramaa Chitale from Elyisa Group, Ltd. (Shanghai, China) for analysis of the data, interpretation of the results, and drafting the manuscript. The data analysis of this study was supported by Sanofi Taiwan Co. Ltd. (Taipei, Taiwan).

Conflicts of Interest: The authors declare no conflict of interest.

\section{References}

1. Berry, A.; Busse, W.W. Biomarkers in asthmatic patients: Has their time come to direct treatment? J. Allergy Clin. Immunol. 2016, 137, 1317-1324. [CrossRef]

2. Asthma, G.I.F. Global Strategy for Asthma Managment and Prevention. 28 August 2020. 2020. Available online: https: //ginasthma.org/wp-content/uploads/2020/06/GINA-2020-report_20_06_04-1-wms.pdf (accessed on 28 August 2020).

3. Tiotiu, A. Biomarkers in asthma: State of the art. Asthma Res. Pract. 2018, 4, 1-10. [CrossRef] [PubMed]

4. Vijverberg, S.J.; Hilvering, B.; Raaijmakers, J.A.; Lammers, J.W.J.; Maitland-van der Zee, A.H.; Koenderman, L. Clinical utility of asthma biomarkers: From bench to bedside. Biol. Targets Ther. 2013, 7, 199.

5. World Health Organization. Asthma. 6 September 2020. 2020. Available online: https://www.who.int/news-room/q-adetail/asthma\#: \{\}:text=Asthma\%20is\%20the \%20most $\% 20$ common, people\%20are $\% 201$ iving $\% 20$ with $\% 20$ asthma (accessed on 28 August 2020).

6. Hsiao, H.J.; Wang, L.C.; Yang, Y.H.; Lee, J.H.; Yu, H.H.; Lin, Y.T.; Chiang, B.L. A nationwide survey of the severity, comorbidity, and mortality of hospitalized patients with asthma in Taiwan. Pediatr. Neonatol. 2013, 54, 254-260. [CrossRef]

7. Lai, C.K.; de Guia, T.S.; Kim, Y.-Y.; Kuo, S.-H.; Mukhopadhyay, A.; Soriano, J.B.; Trung, P.L.; Zhong, N.S.; Zainudin, N.; Zainudin, B. Asthma control in the Asia-Pacific region: The asthma insights and reality in Asia-Pacific study. J. Allergy Clin. Immunol. 2003, 111, 263-268. [CrossRef] 
8. Ma, Y.-C.; Lin, C.-C.; Yang, S.-Y.; Chen, H.-J.; Li, T.-C.; Lin, J.-G. Time trend analysis of the prevalence and incidence of diagnosed asthma and traditional Chinese medicine use among adults in Taiwan from 2000 to 2011: A population-based study. PLoS ONE 2015, 10, e0140318. [CrossRef]

9. Sun, H.-L.; Lue, K.-H. Health care utilization and costs of adult asthma in Taiwan. Allergy Asthma Proc. 2008, $29,177-181$. [CrossRef] [PubMed]

10. Strimbu, K.; Tavel, J.A. What are biomarkers? Curr. Opin. HIV Aids 2010, 5, 463. [CrossRef]

11. Medrek, S.; Parulekar, A.D.; Hanania, N.A. Predictive biomarkers for asthma therapy. Curr. Allergy Asthma Rep. 2017, 17, 69. [CrossRef]

12. Seys, S.F.; Scheers, H.; Brande, P.V.D.; Marijsse, G.; Dilissen, E.; Bergh, A.V.D.; Goeminne, P.C.; Hellings, P.W.; Ceuppens, J.L.; Dupont, L.J.; et al. Cluster analysis of sputum cytokine-high profiles reveals diversity in $\mathrm{T}(\mathrm{h})$ 2-high asthma patients. Respir. Res. 2017, 18, 39. [CrossRef]

13. Peters, M.; Mekonnen, Z.; Yuan, S.; Bhakta, N.R.; Woodruff, P.G.; Fahy, J.V. Measures of gene expression in sputum cells can identify TH2-high and TH2-low subtypes of asthma. J. Allergy Clin. Immunol. 2014, 133, 388-394.e5. [CrossRef]

14. Dunican, E.M.; Fahy, J.V. The role of type 2 inflammation in the pathogenesis of asthma exacerbations. Ann. Am. Thorac. Soc. 2015, 12 (Suppl. 2), S144-S149. [PubMed]

15. Price, D.B.; Bosnic-Anticevich, S.; Pavord, I.D.; Roche, N.; Halpin, D.M.; Bjermer, L.; Usmani, O.S.; Brusselle, G.; Ming, S.W.Y.; Rastogi, S. Association of elevated fractional exhaled nitric oxide concentration and blood eosinophil count with severe asthma exacerbations. Clin. Transl. Allergy 2019, 9, 41. [CrossRef]

16. Saito, J.; Gibeon, D.; Macedo, P.; Menzies-Gow, A.; Bhavsar, P.K.; Chung, K.F. Domiciliary diurnal variation of exhaled nitric oxide fraction for asthma control. Eur. Respir. J. 2014, 43, 474-484. [CrossRef]

17. Wang, Z.; Pianosi, P.; Keogh, K.; Zaiem, F.; Alsawas, M.; Alahdab, F.; Almasri, J.; Mohammed, K.; Larrea-Mantilla, L.; Farah, W.; et al. The Clinical Utility of Fractional Exhaled Nitric Oxide (FeNO) in Asthma Management; Report No.: 17(18)-EHC030-EF; Agency for Healthcare Research and Quality (US): Rockville, ML, USA, 2017.

18. Malinovschi, A.; Janson, C.; Borres, M.; Alving, K. Simultaneously increased fraction of exhaled nitric oxide levels and blood eosinophil counts relate to increased asthma morbidity. J. Allergy Clin. Immunol. 2016, 138, 1301-1308.e2. [CrossRef] [PubMed]

19. Parulekar, A.; Diamant, Z.; Hanania, N.A. Role of T2 inflammation biomarkers in severe asthma. Curr. Opin. Pulm. Med. 2016, 22, 59-68. [CrossRef]

20. Wu, L.C.; Zarrin, A.A. The production and regulation of IgE by the immune system. Nat. Rev. Immunol. 2014, 14, 247-259. [CrossRef] [PubMed]

21. Korevaar, D.A.; Westerhof, G.A.; Wang, J.; Cohen, J.F.; Spijker, R.; Sterk, P.J.; Bel, E.H.; Bossuyt, P.M.M. Diagnostic accuracy of minimally invasive markers for detection of airway eosinophilia in asthma: A systematic review and meta-analysis. Lancet Respir. Med. 2015, 3, 290-300. [CrossRef]

22. Asthma, G.I.F. Global Strategy for Asthma Management and Prevention 2019. Available online: https://ginasthma.org/wpcontent/uploads/2019/06/GINA-2019-main-report-June-2019-wms.pdf (accessed on 28 August 2020).

23. Busse, W.W. Biological treatments for severe asthma: A major advance in asthma care. Allergol. Int. 2019, 68, 158-166. [CrossRef] [PubMed]

24. Castro, M.; Corren, J.; Pavord, I.D.; Maspero, J.; Wenzel, S.; Rabe, K.F.; Busse, W.W.; Ford, L.; Sher, L.; Fitzgerald, J.M.; et al. Dupilumab efficacy and safety in moderate-to-severe uncontrolled asthma. N. Engl. J. Med. 2018, 378, 2486-2496. [CrossRef]

25. Ortega, H.G.; Yancey, S.W.; Mayer, B.; Gunsoy, N.B.; Keene, O.N.; Bleecker, E.R.; Brightling, C.E.; Pavord, I.D. Severe eosinophilic asthma treated with mepolizumab stratified by baseline eosinophil thresholds: A secondary analysis of the DREAM and MENSA studies. Lancet Respir. Med. 2016, 4, 549-556. [CrossRef]

26. Ko, H.K.; Cheng, S.L.; Lin, C.H.; Lin, S.H.; Hsiao, Y.H.; Su, K.C.; Yu, C.J.; Wang, H.C.; Sheu, C.C.; Chiu, K.C.; et al. Blood tryptase and thymic stromal lymphopoietin levels predict the risk of exacerbation in severe asthma. Sci. Rep. 2021, 11, 8425. [CrossRef] [PubMed] 
27. Dougherty, R.H.; Sidhu, S.S.; Raman, K.; Solon, M.; Solberg, O.D.; Caughey, G.H.; Woodruff, P.G.; Fahy, J.V. Accumulation of intraepithelial mast cells with a unique protease phenotype in T (H)2-high asthma. J. Allergy Clin. Immunol. 2010, 125, 1046-1053. [CrossRef] [PubMed]

28. Kraft, M.; Martin, R.J.; Lazarus, S.C.; Fahy, J.V.; Boushey, H.A.; Lemanske, R.F., Jr.; Szefler, S.J. Asthma Clinical Research Network. Asthma Clinical Research Network. Airway tissue mast cells in persistent asthma: Predictor of treatment failure when patients discontinue inhaled corticosteroids. Chest 2003, 124, 42-50. [CrossRef] 